\title{
Quarterly Role of contrast enhanced ultrasound in hepatic ORevieure imaging
}

\author{
Ekta Dhamija and Shashi B Paul
}

\begin{abstract}
Department of Radiodiagnosis, Grey scale ultrasound (US) is the first line imaging modality used for the evaluation of liver by All India Institute of Medical Sciences, New Delhi - 110029, India the radiologists and clinicians worldwide. It is a simple, inexpensive, safe and an easily available technique. US has the ability to delineate the hepatic parenchyma and differentiate the cystic

Correspondence:

Dr. Shashi B Paul

Email:shashi.aiims@gmail.com from solid hepatic lesions. However, it has limited accuracy in the detection and characterization of focal liver lesions (FLL). CEUS is a major breakthrough in ultrasound imaging which evolved with the aim of overcoming these limitations of US. With the use of ultrasound contrast agents(UCAs), CEUS has the ability to detect the intranodular hemodynamics and thereby provide information of the enhancement pattern of the lesion resulting in reliable characterization of the FLL. This capability brings it at par with the cross sectional contrast enhanced imaging techniques of computed tomography and magnetic resonance imaging. UCAs are safe, non-nephrotoxic and thus can be used to evaluate patients with renal failure as well. The technique of CEUS is simple, requires few minutes to perform, portable, lacks ionising radiation and above all is a cost-effective modality. These advantages have made CEUS an established modality for hepatic imaging. Besides detection and characterization of FLL, it also plays a vital role in the management and repeated follow up of treated patients of FLL. Newer clinical applications of CEUS with promising results are also being unravelled .This review highlights the multifaceted role of CEUS in hepatic imaging and its upcoming clinical applications.
\end{abstract}

KEYWORDS: Grey scale ultrasound, contrast enhanced ultrasound, ultrasound contrast agents, focal liver lesions and hepatic imaging

\section{Introduction}

Grey scale ultrasound (US) is the first line imaging modality for evaluation of liver diseases worldwide. This is because, it is readily available, easy to perform, provides rapid diagnosis, is safe, free from ionizing radiation and is cost-effective. ${ }^{1}$ It can delineate hepatic parenchyma extremely well and is excellent at differentiating cystic from solid hepatic lesions. Over the last six decades, diagnostic US has witnessed a plethora of innovative techniques like color Doppler (CD), power Doppler (PDUS), tissue harmonic imaging and contrast enhanced US (CEUS). CD is useful for detection of blood flow within the large vessels but is unable to detect flow perpendicular to the sound field. Moreover, artifacts limit its diagnostic accuracy. The PDUS has low sensitivity for detection of micro-flow in the nodules and is thus not able to pick up intranodular 
hemodynamics' - a pre-requisite for characterizing focal liver lesions (FLL). ${ }^{2}$

Contrast enhanced ultrasound (CEUS) is a major breakthrough in US imaging which evolved to overcome the limitation of lesion characterization by US. As the name implies, this technique uses ultrasound contrast agents (UCAs) for performing US. UCAs are tiny microbubbles of diameter of 110 microns and contain a gas stabilized by a shell. They are pure blood pool agents and remain exclusively in the intravascular compartment providing detailed information of the microvascularity and microperfusion of the lesion in the real-time US aiding in detection and characterization of the FLL. ${ }^{3}$ Currently SonoVue (Bracco, Milan, Italy) is the only UCA available in India. UCAs contain insoluble gases like sulphur hexafluoride $\left(\mathrm{SF}_{6}\right)$ or perfluorocarbons and a surface shell made of different substances like phospholipids, albumin or polymers providing better stabilization. The shell is metabolized by the liver and the gas is eliminated by lungs during respiration. ${ }^{4}$ After intravenous injection, due to the low solubility of UCA in water, better stabilization and strong harmonic response, UCA increases the backscatter of ultrasound producing enhancement of the received signal from the flowing blood. ${ }^{5}$ This results in prolonged visualization of dynamic enhancement of the liver in three distinct phases on real-time ultrasound scanning. ${ }^{6}$

CEUS requires a specific US machine equipped with contrast-specific imaging software based on tissue harmonic and pulse inversion techniques. ${ }^{78}$ These techniques enable visualization of microvascular perfusion in real-time at low mechanical index (MI) with suppression of the background tissue signal. ${ }^{9}$ It also allows quantitative assessment of perfusion of nodules using parametric CEUS. Given these advantages, CEUS has become an important tool for hepatic imaging. This review focuses on applications of CEUS in evaluation of liver lesions.

\section{Technique of CEUS of liver}

CEUS is performed by establishing an intravenous access in the antecubital fossa using a 20 gauge venflon with a three way connector for the injection of UCA (SonoVue). The contrast is prepared in soluble form 5 minutes prior to commencing study and is shaken well to generate the microbubbles. The FLL is focussed on B mode US and then while maintaining it in the field of view, contrast-specific imaging mode is switched on. A simultaneous display of the tissue and contrast signals can be seen on the monitor as a dual window (option in some machines). SonoVue ( $2.4 \mathrm{ml}$ per nodule) is administered as an intravenous bolus followed by flushing with $10 \mathrm{ml}$ of 0.9 percent normal saline. The enhancement pattern of FLL is observed in real-time by continuously scanning till the three vascular phases postinjection are complete. The arterial phase (AP) lasts from 10-30 secs post injection; the portovenous phase (PV) between 3 to 120 secs and the delayed phase (DP) is measured from 120 secs with intermittent scanning up to 4-5 mins as per requirement. ${ }^{9}$ Observations are recorded in cine mode. Postprocedure, the patient is observed for about half-an-hour for any adverse reaction due to UCA. If a second liver nodule needs to be evaluated in the same patient, a fresh study is performed after 10 minutes with another intravenous contrast bolus. The most recent UCA- Sonazoid (Daiichi-Sankyo, Tokyo, Japan) - has an additional benefit of having the longest window period of imaging during the post-vascular phase called the 'Kupffer phase'. The Kupffer cells phagocytose the Sonazoid microbubbles, thus retaining it in the liver for a long period, allowing prolonged assessment upto 60 minutes. ${ }^{10}$ However, Sonazoid is not available in India so far.

\section{Applications of CEUS in hepatic imaging}

The European Federation of Societies for Ultrasound in Medicine and Biology (EFSUMB) has issued a set of guidelines for the use of CEUS. ${ }^{11}$ These guidelines have been framed with the consensus of other Global Federations of Ultrasound in Medicine and Biology (World, Asian, American, Australasian, Latin-American Federations and International contrast US Society). These universal guidelines have established that CEUS is the first line of imaging modality for liver lesions. Similar to the cross-sectional imaging techniques like CT and MRI, it is now possible to detect and characterize FLL by realtime CEUS.

\section{Detection of focal liver lesions (FLL):}

US has been the imaging modality of choice for detection of FLL despite its lower diagnostic accuracy as compared to CT and MRI. ${ }^{3}$ The sensitivity and specificity further drops in cases with underlying chronic liver disease where the liver is small, shrunken with poor acoustic window, presence of multiple isoechoic regenerative nodules as part of cirrhosis and limitation of detection of tiny nodules less than a centimeter. 
CEUS has completely transformed the role of US in FLL detection with improved diagnostic accuracy from $27-42 \%$ with grey scale US to $87-89 \%$ with CEUS. ${ }^{12,13}$ Various studies have shown that CEUS enhances the efficacy of gray-scale US ${ }^{14-19}$ (Table 1). Numerous studies have demonstrated the superiority of CEUS over CT in this regard and a comparable accuracy of the two at overall performance. ${ }^{20,21}$ If a proper acoustic window is available, the sensitivity, specificity and accuracy of CEUS improves even further to $94.6 \%, 87.5 \%$ and $93.3 \%$, respectively. ${ }^{22}$

\section{Characterization of FLL}

For differentiating a benign from malignant nodule, it is extremely important to obtain the clinical history of the patient and to look for underlying cirrhosis as the type and frequency of the tumor varies in cirrhotic and non-cirrhotic liver. The enhancement pattern of the FLL is assessed in relation to the surrounding hepatic parenchyma; hyperenhancing - indicating more enhancement, hypoenhancing - if it is less enhancing and isoenhancing - if it has similar enhancement as the adjoining liver parenchyma. The assessment of enhancement characteristics of the nodule in three phases of enhancement, namely AP, VP and the DP are important. It is the DP which is most crucial in differentiating between benign and malignant nodules whereas AP helps in predicting the histology of the tumor. $^{3,17,18,23-25}$

CEUS has high diagnostic accuracy of $80-95 \%$ for characterization of benign and malignant FLLs. ${ }^{26} \mathrm{~A}$ hypoenhancing nodule in DP phase is the hallmark of a malignant nodule. This is due to the fact that the malignant nodule is devoid of hepatic sinusoids and the UCA fails to accumulate within it and thus the nodule is seen as hypoenhancing (dark defect) during DP. ${ }^{27}$ This feature of hypoenhancement during DP has a positive predictive value (PPV) of $86.4 \%$ and a negative predictive value (NPV) of $38 \% .^{16}$ Benign lesions on the contrary remain isoenhancing to hyperenhancing in the PV and DP because the UCAs tend to get retained in the normal healthy liver due to slow flow through the sinusoids. Almost $86 \%$ to $93 \%$ of benign lesions retain contrast and are hyperenhancing in the late phase, while $78 \%$ to $98 \%$ of malignant lesions depict washout (hypoenhancement) in the DP of CEUS ${ }^{3,23,24}$ (Figures 1and 2).

\section{a. CEUS patterns of benign FLL}

Various benign FLL depict classic enhancement patterns, quite similar to the type seen on contrast CT and MRI. Table 2 illustrates the various patterns in greater detail.

Hemangioma is the most common benign FLL with a prevalence of 1-20\% ${ }^{28} \mathrm{CEUS}$ detects hemangiomas with a high sensitivity and specificity of $96.3 \%$ and $97.5 \%$, respectively. ${ }^{25}$ The characteristic pattern seen in 52-88\% of cases is peripheral nodular enhancement during AP and gradual central filling during PV and DP. Sustained enhancement during DP is seen in about $83-100 \%$ cases $^{17,24,25,29}$ (Figure 1). Small hemangiomas $(<15 \mathrm{~mm})$ may show atypical patterns of rapid homogeneous enhancement on CT/MRI, raising suspicion during diagnosis. CEUS can pick up early peripheral enhancement in the initial 812 seconds in these small, rapidly enhancing masses making the diagnosis more than clear. ${ }^{14}$

Focal nodular hyperplasia (FNH) is the second most common benign hepatic tumor with a prevalence of $0.4-3 \% .^{30} \mathrm{It}$ is considered to be a hepatic pseudo-tumor, developing in response to underlying vascular malformations. CEUS has a higher sensitivity than CT/MRI in diagnosing FNH. ${ }^{14}$ CEUS depicts a hypervascular tumor showing early hyperenhancement during AP with homogeneous enhancement in PV and becomes isoenhancing during DP owing to the presence of portal venous supply. This enhancement pattern is seen in $80 \%$ of FNHs. ${ }^{31}$ The classical spoke-wheel pattern of enhancement radiating from the central vessel with centrifugal homogeneous enhancement of lesion occurs in $95 \%$ of cases with lesion size of at least $3 \mathrm{~cm}$ diameter and in $20-30 \%$ of cases if the lesion is smaller. ${ }^{18,32}$

Hepatic adenoma (HA) is a rare, benign hepatic tumor with

Table 1: Studies comparing conventional US with contrast enhanced ultrasound (CEUS) for diagnosis of focal liver lesions (FLL)

\begin{tabular}{|c|c|c|c|c|c|c|c|}
\hline \multirow[t]{2}{*}{ Author } & \multirow[t]{2}{*}{ Study/ Lesion } & \multicolumn{2}{|c|}{ Sensitivity (\%) } & \multicolumn{2}{|c|}{ Specificity (\%) } & \multicolumn{2}{|c|}{ Accuracy (\%) } \\
\hline & & US & CEUS & US & CEUS & US & CEUS \\
\hline Trillaud et al $2009^{14}$ & 123 patients/ FLL & 40 & 98.2 & 36.8 & 88.2 & 38.2 & 92.7 \\
\hline Albrecht et al $* 2003^{15}$ & 123 patients/ (only metastases) & 94 & 98 & 60 & 88 & - & - \\
\hline Chami et al $2008^{16}$ & 116 patients/ FLL & 58.8 & 68.7 & 50.7 & 67 & - & - \\
\hline Leen et al $2006^{17}$ & $152 \mathrm{FLL}$ & $28.1-59.8$ & $90.2-95.4$ & $30.6-34.6$ & $80.8-89.8$ & $30.6-41.2$ & $86.6-91.2$ \\
\hline Quaia et al $2004^{18}$ & 452 FLL & $52-54$ & $81-85$ & $40-43$ & 95 & $49-51$ & $85-88$ \\
\hline
\end{tabular}

*per patient sensitivity and specificity 
Table 2: Enhancement patterns of benign focal liver lesions ${ }^{11}$

\begin{tabular}{|c|c|c|c|}
\hline Lesion & Arterial phase & Portal venous phase & Late phase \\
\hline \multicolumn{4}{|l|}{ A. Noncirrhotic liver } \\
\hline \multicolumn{4}{|l|}{ Hemangioma } \\
\hline - Typical features & Peripheral nodular enhancement & $\begin{array}{l}\text { Partial/complete centripetal } \\
\text { fill in }\end{array}$ & Complete enhancement \\
\hline - Additional features & $\begin{array}{l}\text { Small lesion: complete, rapid centripetal } \\
\text { enhancement }\end{array}$ & & Nonenhancing regions \\
\hline \multicolumn{4}{|c|}{ Focal nodular hyperplasia } \\
\hline - Typical features & $\begin{array}{l}\text { Hyperenhancing from the center, complete, } \\
\text { early }\end{array}$ & Hyperenhancing & Iso/Hyperenhancing \\
\hline $\begin{array}{l}\text { - Additional features } \\
\text { Hepatocellular aden }\end{array}$ & Spoke-wheel arteriesFeeding artery & Unenhanced central scar & Unenhanced central scar \\
\hline $\begin{array}{l}\text { - Typical features } \\
\text { - Additional features }\end{array}$ & $\begin{array}{l}\text { Hyperenhancing, complete } \\
\text { Non enhancing regions }\end{array}$ & $\begin{array}{l}\text { Isoenhancing } \\
\text { Hyperenhancing } \\
\text { Nonenhancing regions }\end{array}$ & $\begin{array}{l}\text { Isoenhancing } \\
\text { Slightly hypoenhancing } \\
\text { Nonenhancing regions }\end{array}$ \\
\hline $\begin{array}{l}\text { Focal fatty infiltrati } \\
\text { - Typical features } \\
\text { Focal fatty sparing }\end{array}$ & Isoenhancing & Isoenhancing & Isoenhancing \\
\hline $\begin{array}{l}\text { - Typical features } \\
\text { Abscess }\end{array}$ & Isoenhancing & Isoenhancing & Isoenhancing \\
\hline - Typical features & $\begin{array}{l}\text { Peripheral enhancement, no central } \\
\text { enhancement }\end{array}$ & $\begin{array}{l}\text { Hyper-/isoenhancing rim, no } \\
\text { central enhancement }\end{array}$ & $\begin{array}{l}\text { Hypoenhancing rim, no } \\
\text { central enhancement }\end{array}$ \\
\hline - Additional features & $\begin{array}{l}\text { Enhanced septa } \\
\text { Hyperenhanced liver segment }\end{array}$ & $\begin{array}{l}\text { Hypoenhancing rim } \\
\text { Enhanced septa } \\
\text { Hyperenhanced liver segment }\end{array}$ & \\
\hline Simple cyst & & & \\
\hline - Typical features & Nonenhancing & Nonenhancing & Nonenhancing \\
\hline B. Cirrhotic liver & & & \\
\hline Regenerative nodule & pplastic) & & \\
\hline $\begin{array}{l}\text { - Typical features } \\
\text { (not diagnostic) }\end{array}$ & Isoenhancing & Isoenhancing & Isoenhancing \\
\hline - Additional features & Hypoenhancing & & \\
\hline
\end{tabular}

In cirrhotic liver simple cysts, hemangiomas and abscesses may also be found and show the same enhancement pattern as in noncirrhotic livers. All other entities are rare findings in cirrhotic livers.
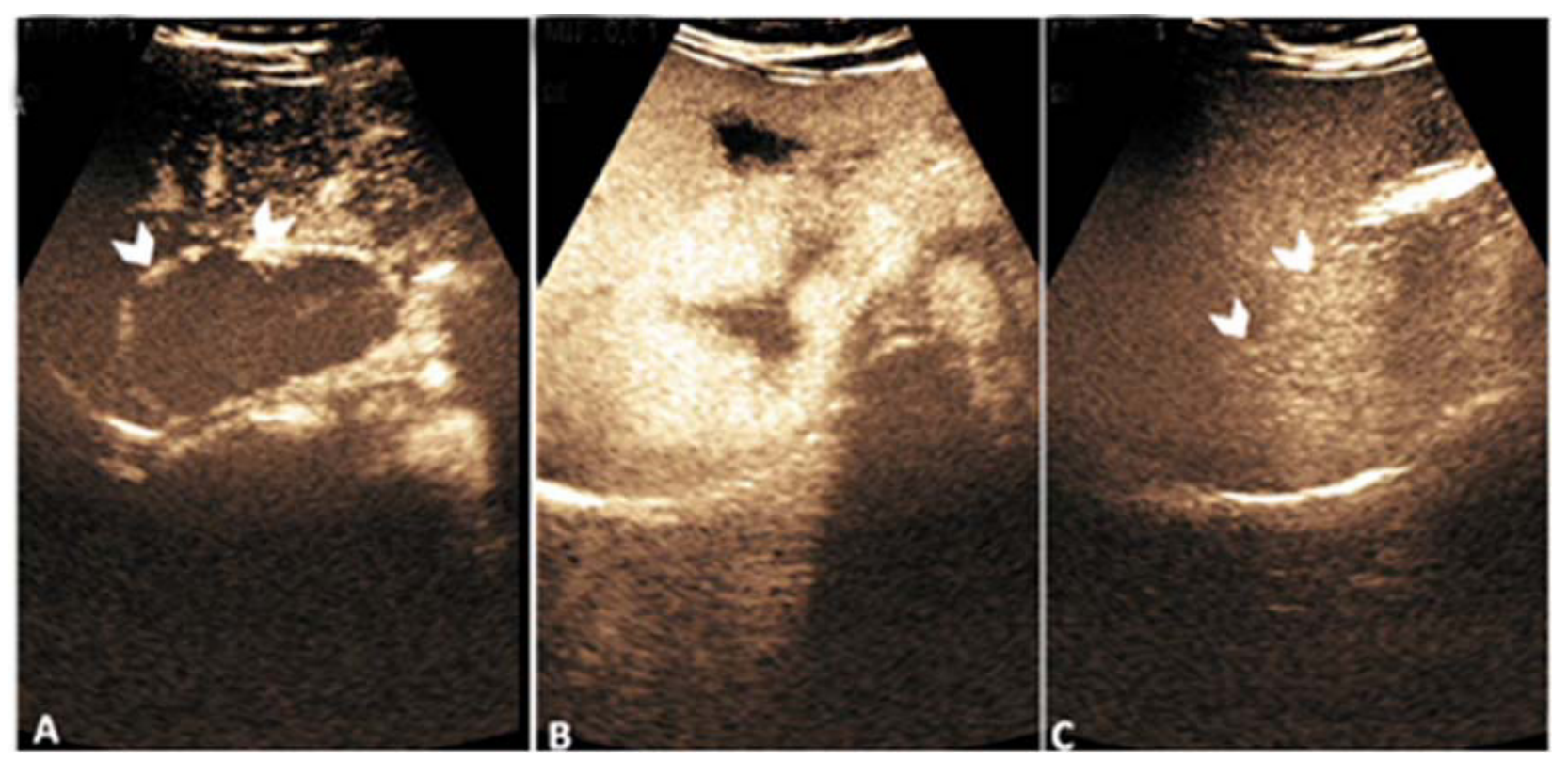

Figure 1: Classical pattern of a benign FLL on CEUS. (A) A lobulated mass in segment 7 of liver showing peripheral nodular enhancement (arrow heads) in the AP ( 13 seconds) with (B) gradual fill-in of contrast during PV phase (148 seconds) and retention of contrast in DP at 344 seconds (arrowheads) (C) suggestive of hemangioma 
an incidence of $0.03 \%$. It is composed of atypical hepatocytes devoid of Kupffer cells. ${ }^{32}$ It is frequently seen in females of reproductive age group and is associated with intake of oral contraceptives, obesity and metabolic syndrome. ${ }^{33}$ HA shows non-specific imaging characteristics on US and variable enhancement patterns on CEUS. Small adenomas may show homogeneous enhancement but larger tumors have heterogeneous enhancement due to presence of hemorrhage and necrosis within, the later showing no enhancement on any imaging modality. Centripetal enhancement due to presence of multiple thin arteries predominantly in the periphery of the lesion has been described for HA on CEUS. ${ }^{9}$ HA may show hypoenhancement in DP as it has no portal venous supply and in such a situation it may be very difficult to differentiate it from HCC. In such a scenario, clinical history of chronic oral contraceptive use and normal level of serum alpha-fetoprotein can help clinch the diagnosis.

Hepatic abscesses are most commonly of bacterial etiology. CEUS characteristics are quite similar to US findings, varying with the stage of abscess. Early abscess is solid and shows diffuse heterogeneous enhancement in the AP persisting in the PP and LP. A transient peri-lesional enhancement also occurs during AP which in very few cases may show hypoenhancement in the PP. ${ }^{34}$ This pattern of the abscess needs to be differentiated from a necrotic metastasis which appears as a punched-out enhancing defect in the DP, while the abscess depicts as an ill-defined area of hypoenhancement. With maturity, the abscess develops fluid within and is seen as an enhancing rim with internal septations on CEUS.

Hepatic cysts are best evaluated by US. Cysts are seen as thin walled anechoic lesions with no enhancement on any phase of CEUS

Focal fatty change includes focal fatty infiltration or fatty sparing within the liver parenchyma. These occur in typical locations like, adjacent to the falciform ligament, portal vein or the gall bladder. It has geographical margins with no mass effect and normal hepatic vessels can be seen coursing through. When seen in atypical locations, these may mimic malignancy. On CEUS, they show similar enhancement as the liver parenchyma in all three phases with normal vascular channels coursing through them and thus can be differentiated from FLL. ${ }^{35}$

\section{b. CEUS patterns of malignant FLL}

Compared to CT/MRI, CEUS has a better sensitivity (98.2\% vs $68.5 \%$ ), specificity ( $88.1 \%$ vs $74.6 \%$ ) and a diagnostic accuracy of $92.6 \%$ vs $71.9 \%$ in characterizing malignant liver lesions. ${ }^{14}$ CEUS offers a multifaceted tool for patients with malignant liver disease ranging from differentiating between different stages of hepatocarcinogenesis to diagnosis of frank malignant

Table 3: Enhancement patterns of malignant focal liver lesions ${ }^{11}$

\begin{tabular}{|c|c|c|c|}
\hline Lesion & Arterial phase & Portal venous phase & Late phase \\
\hline \multicolumn{4}{|l|}{ A. Noncirrhotic liver } \\
\hline \multicolumn{4}{|l|}{ Metastasis } \\
\hline - Typical features & Rim-enhancement & Hypoenhancing & Hypo/nonenhancing \\
\hline - Additional features & $\begin{array}{l}\text { Complete enhancement } \\
\text { Hyperenhancement } \\
\text { Nonenhancing regions }\end{array}$ & Nonenhancing regions & Nonenhancing regions \\
\hline \multicolumn{4}{|l|}{ HCC } \\
\hline - Typical features & Hyperenhancing & Isoenhancing & Hypo/nonenhancing \\
\hline - Additional features & Nonenhancing regions & Nonenhancing regions & Nonenhancing regions \\
\hline \multicolumn{4}{|l|}{ Cholangiocarcinoma } \\
\hline - Typical features & $\begin{array}{l}\text { Rim-like hyperenhancement, } \\
\text { central hypoenhancement }\end{array}$ & Hypoenhancing & Nonenhancing \\
\hline \multirow[t]{3}{*}{ - Additional features } & Nonenhancing regions & & \\
\hline & Inhomogeneous & & \\
\hline & Hyperenhancement & Nonenhancing regions & Nonenhancing regions \\
\hline \multicolumn{4}{|l|}{ B. Cirrhotic liver } \\
\hline \multicolumn{4}{|l|}{ HCC } \\
\hline \multirow[t]{2}{*}{ - Typical features } & Hyperenhancing, complete & Isoenhancing & Hypoenhancing \\
\hline & Nonenhancing areas (if large) & Nonenhancing regions & (slightly or moderately) \\
\hline \multirow[t]{3}{*}{ - Additional features } & Basket pattern, chaotic vessels & Nonenhancing & Isoenhancing \\
\hline & Enhancing tumor thrombus & & Nonenhancing \\
\hline & Hypo/nonenhancing & & \\
\hline
\end{tabular}

Explanation: Other malignancies in cirrhosis have the same patterns as in noncirrhotic livers 
nodules, treatment guidance for locoregional therapeutic options for HCC and post-treatment response assessment. CEUS patterns of malignant FLL are detailed in Table 3.

\section{c. Multi-step hepatocarcinogenesis in a cirrhotic liver}

Regenerative nodules (RN) are commonly supplied by the normal hepatic artery and the portal vein. As the process progresses towards malignancy, through the dysplastic nodule (DN) followed by frank hepatocellular carcinoma (HCC), abnormal arterial supply increases and normal arterial and portal supply decreases. ${ }^{36}$ Hence these nodules have variable characteristics and invariably pose a diagnostic dilemma. On CEUS, most RN and DN do not show any enhancement (isoenhancing) in any phase. At times they may show transient hypoenhancement during AP. DN may have variable and inconsistent arterial and portal supplies or significant overlap of vascular supply, leading to problems in diagnosis with all modalities (CEUS, CT, MRI). Due to continuous real-time scanning by CEUS, it is possible to pick up subtle, transient enhancement patterns in these nodules which help differentiate HCC from DN. ${ }^{36}$

Hepatocellular carcinoma is the most common primary hepatic malignancy and more than $80-90 \%$ of cases occur on the background of chronic viral liver disease.$^{37}$ Early diagnosis and treatment are critical for increasing survival. ${ }^{29} \mathrm{CEUS}$ detects HCC with a very high sensitivity of $100 \%$, specificity of $95.6 \%$, PPV of $66.7 \%$ and NPV of $100 \%$ in comparison to the gold standards of histopathology and CT/MRI. ${ }^{38}$ In a multicentric study ${ }^{14}$ comparing CEUS with CT and/or dynamic MRI for nodule characterization, CEUS proved to be $30.2 \%$ more sensitive in diagnosing malignancy and $16.1 \%$ more specific in excluding malignancy with an overall accuracy of $22.9 \%$. When compared with histology, CEUS showed higher sensitivity (95.5\%) and specificity (75\%), and was likewise better than CT (72.2\% and $37.5 \%$, respectively) and MRI (81.8\% and $42.9 \%$, respectively).

HCC exhibits typical enhancement patterns on CEUS by virtue of being a highly vascular tumor. More than $90 \%$ of HCC are hyperenhancing in AP (arterialisation) and 83-97\% show washout (hypoenhancement) during DP ${ }^{29}$ (Figure 2). However, the pattern of washout in DP may vary with the histology of HCC; more the differentiation, slower is the wash out. Poorly differentiated HCC show early washout than moderately and well differentiated HCC. ${ }^{39,40}$ Moreover, well differentiated HCC may show retained contrast in the DP which washes out after a long duration. In such a situation, it becomes mandatory to

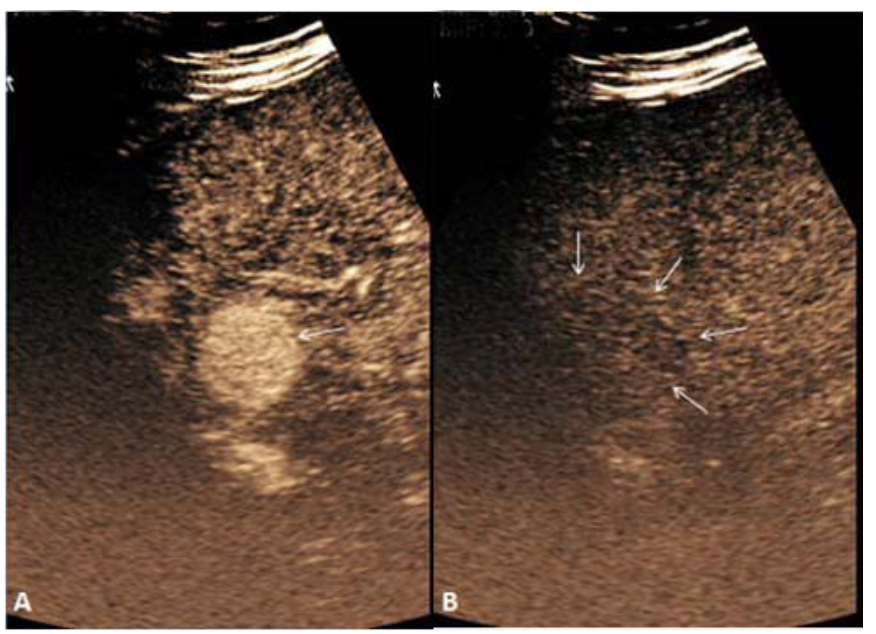

Figure 2: Classical pattern of a malignant FLL on CEUS (A) AP image at 20 seconds showing a homogenously enhancing mass in right lobe of liver with washout (arrows) in the DP at 192 seconds suggestive of HCC (B)

observe the nodule for longer duration in order to detect the delayed washout. Despite this manoeuvre, about half of well differentiated HCCs may not show washout. ${ }^{41}$ Any nodule depicting an atypical pattern on screening for cirrhosis should be considered highly suspicious and should be further investigated by fine needle aspiration or biopsy to rule out HCC. ${ }^{42}$ CEUS is superior to CT and MRI in demonstrating HCC hypervascularity (arterialisation). ${ }^{14}$ During CEUS it is mandatory to evaluate the portal vein. A malignant portal vein thrombus shows heterogeneous enhancement or irregular linear vessels on CEUS, while a bland thrombus tends to be avascular. $^{43}$

Intrahepatic cholangiocarcinoma (ICC) is the second most common hepatic malignancy arising from the biliary tract. ICC has a variable pattern of enhancement. Characteristically, ICC demonstrates peripheral rim enhancement in AP with hypoenhancement during PV and DP. ${ }^{44,45}$ In a study of 21 pathologically proven ICC, $52.4 \%$ of ICC displayed peripheral enhancement and $47.6 \%$ showed homogeneous enhancement in AP, with contrast washout in DP. ${ }^{45}$ This is in contrast to the pattern on CT and MRI, where ICC demonstrates gradual contrast enhancement during DP. This is largely because, UCA is confined to the intravascular compartment and does not cross the vascular endothelium to enter the extracellular/ interstitial space. Thus there is no enhancement of the nodule during DP. ICC on the background of liver cirrhosis is rare but could mimic HCC on CEUS and may require confirmation with alternate imaging modality or biopsy. ${ }^{45}$

Metastases of the liver are fairly common and is the commonest indication for performing US. CEUS has a sensitivity, 
specificity, PPV and NPV of 93.1\%, 100\%, 100\% and 91.3\%, respectively, in diagnosing liver metastases. ${ }^{38}$ Multiple enhancement patterns are seen in metastases depending upon the primary malignancy. Peripheral rim or diffuse enhancement in AP with rapid washout during PV and DP (in less than 60 secs) is the commonest pattern. ${ }^{42}$ Hypervascular metastases may show homogeneous enhancement on AP and mimic HCC. In patients with known primary malignancy, diagnosis of metastases can be established easily but a detailed clinical evaluation and serum alpha-fetoprotein levels are required for a confident diagnosis in the absence of relevant history.

\section{CEUS guidance for interventional procedures}

CEUS plays a crucial role in guiding diagnostic and therapeutic interventions for tumors not detected by US. It outlines the margins of the tumor and extent of malignant infiltration well. Thus, accurate intratumoral needle placement can be done producing better outcomes. CEUS is the sole imaging modality which can be safely undertaken in patients of HCC having concomitant renal failure because the contrast used in not nephrotoxic.

\section{Assessment of post treatment response}

So far, CT/ MRI have been the gold standards for monitoring treatment response to locoregional therapies including transarterial chemoembolization (TACE) and percutaneous ablative therapies like radiofrequency ablation (RFA), ethanol (PEI) or acetic acid ablation (PAI) and microwave/laser ablation. However, CT/ MRI pose limitations of cost, radiation exposure and limited resolution. CEUS overcomes these limitations while maintaining comparable efficiency. Recently anti-angiogenic drugs that help in reducing tumor neo-vascularity have come into use for treating HCC. CEUS is extremely useful in assessing treatment response in such cases by assessing reduction or absence of intratumoral vascularity. ${ }^{46,47}$

Ablative therapies like RFA, PEI and PAI are routinely performed under US guidance to effect coagulation necrosis of the tumour. Majority of recurrent or residual masses tend to occur adjacent to ablated tissue and are usually small in size. US is unable to differentiate between the viable and non-viable tissue. CEUS however can reliably differentiate residual/ recurrent tumour tissue even immediately after ablation. The residual viable tissue is seen as a hyperenhancing tissue within the tumour during AP with washout in PP and DP, while the non-viable tissue is hypoenhancing in all the phases (Figure 3). CEUS has a sensitivity of $87 \%$ and specificity of 98.4\% following loco-regional therapies of TACE, RFA and PEI. ${ }^{48}$ It has also been seen that RFA performed under CEUS guidance produces better results and lesser number of repeat treatment sessions. ${ }^{49}$

TACE is a commonly used treatment for unresectable HCC. ${ }^{50,51}$ It involves administration of a lipid-drug suspension (lipiodol) within the tumor bed by selectively cannulating the feeding artery trans-arterially. This lipiodol is radio-dense and persists at the tumor site for many months. On post TACE CT, the high attenuation caused by lipiodol tends to obscure the underlying tumor making it difficult to assess the tumor response. Due to these limitations, MRI is considered the gold standard. However, MRI has its own limitations like limited availability, reduced sensitivity for detection of small lesions, influence of motion artifacts and above all the cost. ${ }^{52}$ CEUS has a higher detection rate for residual disease compared to MPCT despite the presence of lipiodol in patients of TACE ${ }^{53}$ (Figure 4). Due to this, CEUS has been included as a modality in the Response Evaluation Criteria in Solid Tumors (RECIST) guidelines for HCC. These guidelines defined complete response following therapy as the complete disappearance of any previously visualized intralesional enhancement on CEUS. ${ }^{54}$

Parametric CEUS is an emerging technique for quantitative assessment of tumor vascularity in residual disease after locoregional or oral chemotherapy of HCC. The perfusion of the tumor in terms of time to peak (TTP), estimated perfusion

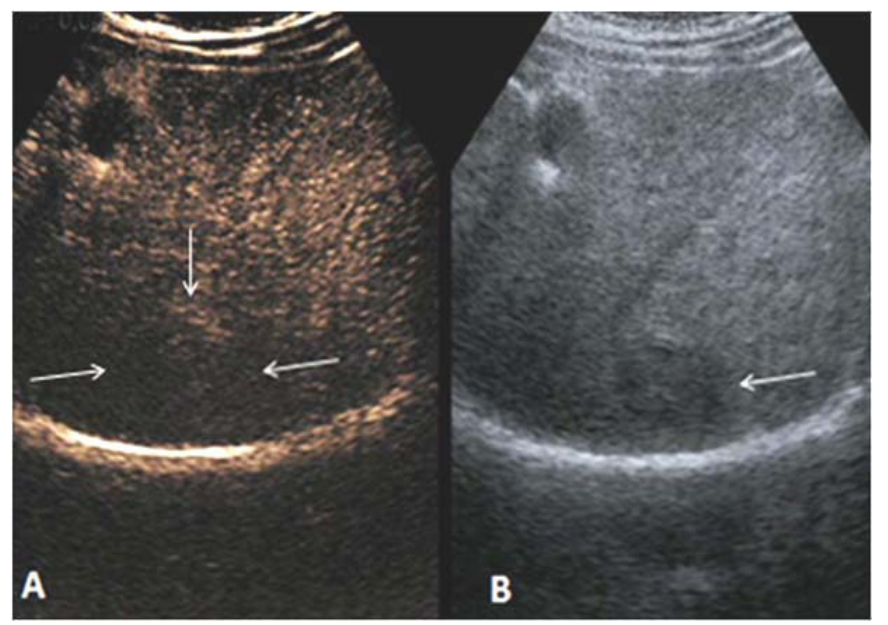

Figure 3: Complete response following acetic acid ablation of segment VII HCC: Simultaneous US display images of contrast and tissue signal (A \& B) showing a well defined hypoenhancing lesion (arrows) with no enhancing viable tissue seen within the treated mass in the AP at 34 seconds (A) suggestive of complete response post ablation 


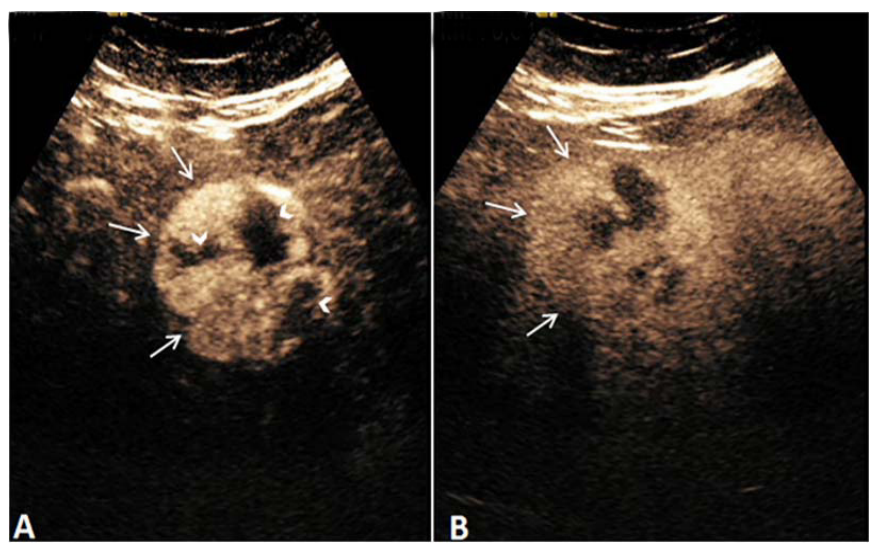

Figure 4: Residual disease post TACE on CEUS performed at one month in a patient of HCC. (A) AP image at 12 seconds showing a heterogenously enhancing mass with areas of enhancement (arrows) depicting residual tumor and non enhancing areas (arrow heads) suggesting necrosis. The enhancement reduces in intensity in the PV at 118 seconds (arrows) (B)

(EP), peak intensity (PI) and area under the time-intensity curve (AUC) can be estimated based on the density of microvessels within the tumor. ${ }^{55}$

\section{Evolving applications of CEUS}

CEUS has been used as a non-invasive technique for assessment of portal hypertension. It measures the arrival time of contrast microbubbles in the hepatic veins (HVAT), which correlates inversely to the degree of liver disease. Reduced HVAT suggests increased severity of liver disease. A strong correlation with hepatic vein pressure gradient (HVPG) makes HVAT a potential alternative to HVPG ${ }^{56}$ Hepatic vein transit time (HVTT) can also be used to differentiate mild hepatitis from cirrhosis. HVTT may complement liver biopsy or serve as an alternative when biopsy is contraindicated. ${ }^{57}$

CEUS has been tried in liver transplant patients for assessing vascular complications like hepatic artery, portal vein, hepatic veins and inferior vena cava thrombosis. Although color Doppler is the modality of choice for large vessel evaluation but post-operative edema precludes its use. Moreover shifting an unstable post-surgical patient for CT/ MRI can be risky. CEUS comes to rescue for such patients with good results. ${ }^{11}$

CEUS can also be useful in hepatic trauma. Though CT is the standard modality, CEUS offers the advantage of immediate bed-side detection of hemoperitoneum and liver laceration. Normal liver is homogeneously hyperechoic during PV and DP of CEUS. The traumatic area (lacerations or contusions) are devascularised and show absent or reduced perfusion which is better appreciated during DP. ${ }^{58}$ US is highly sensitive (63$99 \%$ ) in detecting intra-abdominal fluid, but is relatively inferior at detecting solid organ injury (41\% sensitivity). ${ }^{59-62}$ CEUS improves the sensitivity, specificity, PPV and NPV of conventional US at detecting solid organ injuries in hemodynamically stable patients: $91.4 \%$ vs. $45.7 \%, 100 \%$ vs. $91.8 \%, 100 \%$ vs. $84.2 \%$ and $92.5 \%$ vs. $64.1 \%$, respectively. ${ }^{63}$ Patients with solid organ injury detected on CT can be safely followed-up by CEUS. ${ }^{63}$ Intra-operative CEUS has been used to accurately define the completeness of tumor resection in cases of HCC and liver metastases. It can reliably detect viable tumor tissue in the surgical cuff influencing the aggressiveness of surgery. ${ }^{64,65}$

CEUS is a safe technique. Life threatening complications are extremely rare $(0.001 \%) .{ }^{66}$ Since UCAs are not excreted via the kidney, they can be safely used in patients with renal failure ${ }^{67}$ Portability of CEUS especially for sick patients is highly advantageous. These advantages have made CEUS a preferred modality for various clinical applications. However, CEUS also has a number of limitations. Poor penetration of sound to deeper tissues makes it difficult to assess deep seated nodules (depth $>10 \mathrm{cms}$ ), sub-diaphragmatic lesions and very small lesions $(<1 \mathrm{~cm})$. CEUS has limitation of staging HCC as the entire liver cannot be assessed during the short span of AP. If more nodules are to be studied, repeat contrast injections are required which defeats the purpose of cost effectiveness.

\section{Conclusion}

CEUS has revolutionized the role of US in clinical practice. It has become an established modality for hepatic imaging and offers high diagnostic accuracy for the detection and characterization of FLL, guidance of interventional procedures and assessment of post-treatment response. Newer applications of CEUS are being explored for hepatic disease with promising results.

\section{References}

1. Sahani DV, Kalva SP. Imaging the liver. Oncologist. 2004;9:385-97.

2. Kim TK, Han JK, Kim AY, Choi BI. Limitations of characterization of hepatic hemangiomas using a sonographic contrast agent (Levovist) and power Doppler ultrasonography. $J$ Ultrasound Med. 1999;18:737-43.

3. Wilson SR, Burns PN. An algorithm for the diagnosis of focal 
liver masses using microbubble contrast-enhanced pulse-inversion sonography. AJR Am J Roentgenol. 2006;186:1401-12.

4. Dietrich CF, Cui XW, Boozari B, Hocke M, Ignee A. Contrastenhanced ultrasound (CEUS) in the diagnostic algorithm of hepatocellular and cholangiocellular carcinoma, comments on the AASLD guidelines. Ultraschall Med. 2012;33 Suppl 1:S57-66.

5. Claudon M, Cosgrove D, Albrecht T, Bolondi L, Bosio M, Calliada F, et al. Guidelines and good clinical practice recommendations for contrast enhanced ultrasound (CEUS) - update 2008. Ultraschall Med. 2008;29:28-44.

6. Xu HX. Contrast-enhanced ultrasound: The evolving applications. World J Radiol. 2009;1:15-24.

7. Shapiro RS, Wagreich J, Parsons RB, Stancato-Pasik A, Yeh HC, Lao R. Tissue harmonic imaging sonography: evaluation of image quality compared with conventional sonography. AJR Am J Roentgenol. 1998;171:1203-6.

8. Wilson SR, Greenbaum LD, Goldberg BB. Contrast-enhanced ultrasound: what is the evidence and what are the obstacles? AJR Am J Roentgenol. 2009;193:55-60.

9. Jang JY, Kim MY, Jeong SW, Kim TY, Kim SU, Lee SH, et al. Current consensus and guidelines of contrast enhanced ultrasound for the characterization of focal liver lesions. Clin Mol Hepatol. 2013;19:1-16.

10. Yanagisawa K, Moriyasu F, Miyahara T, Yuki M, Iijima H. Phagocytosis of ultrasound contrast agent microbubbles by Kupffer cells. Ultrasound Med Biol. 2007;33:318-25.

11. Claudon M, Dietrich CF, Choi BI, Cosgrove DO, Kudo M, Nolsoe $\mathrm{CP}$, et al. Guidelines and good clinical practice recommendations for contrast enhanced ultrasound (CEUS) in the liver-update 2012: a WFUMB-EFSUMB initiative in cooperation with representatives of AFSUMB, AIUM, ASUM, FLAUS and ICUS. Ultraschall Med. 2013;34:11-29.

12. Bartolotta TV, Taibbi A, Galia M, Runza G, Matranga D, Midiri M, et al. Characterization of hypoechoic focal hepatic lesions in patients with fatty liver: diagnostic performance and confidence of contrast-enhanced ultrasound. Eur Radiol. 2007;17:650-61.

13. Dai Y, Chen MH, Yin SS, Yan K, Fan ZH, Wu W, et al. Focal liver lesions: can SonoVue-enhanced ultrasound be used to differentiate malignant from benign lesions? Invest Radiol. 2007;42:596-603.

14. Trillaud H, Bruel JM, Valette PJ, Vilgrain V, Schmutz G, Oyen R, et al. Characterization of focal liver lesions with SonoVueenhanced sonography: international multicenter-study in comparison to CT and MRI. World J Gastroenterol. 2009;15:3748-56.

15. Albrecht T, Blomley MJ, Burns PN, Wilson S, Harvey CJ, Leen E, et al. Improved detection of hepatic metastases with pulseinversion US during the liver-specific phase of SHU 508A: multicenter study. Radiology. 2003;227:361-70.

16. Chami L, Lassau N, Malka D, Ducreux M, Bidault S, Roche A, et al. Benefits of contrast-enhanced sonography for the detection of liver lesions: comparison with histologic findings. AJR Am J Roentgenol. 2008;190:683-90.

17. Leen E, Ceccotti P, Kalogeropoulou C, Angerson WJ, Moug SJ, Horgan PG. Prospective multicenter trial evaluating a novel method of characterizing focal liver lesions using contrast-enhanced sonography. AJR Am J Roentgenol. 2006;186:1551-9.

18. Quaia E, Calliada F, Bertolotto M, Rossi S, Garioni L, Rosa L, et al. Characterization of focal liver lesions with contrast-specific US modes and a sulfur hexafluoride-filled microbubble contrast agent: diagnostic performance and confidence. Radiology. 2004;232:420-30.

19. Wilson SR, Jang HJ, Kim TK, Burns PN. Diagnosis of focal liver masses on ultrasonography: comparison of unenhanced and contrast-enhanced scans. J Ultrasound Med. 2007;26:775-87.

20. Hohmann J, Albrecht T, Hoffmann CW, Wolf KJ. Ultrasonographic detection of focal liver lesions: increased sensitivity and specificity with microbubble contrast agents. Eur J Radiol. 2003;46:147-59.

21. Solbiati L, Tonolini M, Cova L, Goldberg SN. The role of contrastenhanced ultrasound in the detection of focal liver leasions. Eur Radiol. 2001;11 Suppl 3:E15-26.

22. Ryu SW, Bok GH, Jang JY, Jeong SW, Ham NS, Kim JH, et al. Clinically useful diagnostic tool of contrast enhanced ultrasonography for focal liver masses: comparison to computed tomography and magnetic resonance imaging. Gut Liver. 2014;8:292-7.

23. Nicolau C, Vilana R, Catala V, Bianchi L, Gilabert R, Garcia A, et al. Importance of evaluating all vascular phases on contrastenhanced sonography in the differentiation of benign from malignant focal liver lesions. AJR Am J Roentgenol. 2006;186:158-67.

24. von Herbay A, Vogt C, Willers R, Haussinger D. Real-time imaging with the sonographic contrast agent SonoVue: differentiation between benign and malignant hepatic lesions. J Ultrasound Med. 2004;23:1557-68.

25. Ding H, Wang WP, Huang BJ, Wei RX, He NA, Qi Q, et al. Imaging of focal liver lesions: low-mechanical-index real-time ultrasonography with SonoVue. J Ultrasound Med. 2005;24:285-97.

26. Tranquart F, Le Gouge A, Correas JM, Ladam Marcus V, Manzoni $\mathrm{P}$, Vilgrain V, et al. Role of contrast-enhanced ultrasound in the blinded assessment of focal liver lesions in comparison with MDCT and CEMRI: Results from a multicentre clinical trial. Eur J Cancer. 2008;6(11) Suppl:9-15.

27. Kono Y, Steinbach GC, Peterson T, Schmid-Schonbein GW, Mattrey RF. Mechanism of parenchymal enhancement of the liver with a microbubble-based US contrast medium: an intravital microscopy study in rats. Radiology. 2002;224:253-7.

28. Mergo PJ, Ros PR. Benign lesions of the liver. Radiol Clin North Am. 1998;36:319-31.

29. Morin SH, Lim AK, Cobbold JF, Taylor-Robinson SD. Use of second generation contrast-enhanced ultrasound in the assessment of focal liver lesions. World J Gastroenterol. 2007;13:5963-70.

30. Maillette de Buy Wenniger L, Terpstra V, Beuers U. Focal nodular hyperplasia and hepatic adenoma: epidemiology and pathology. Dig Surg. 2010;27:24-31.

31. Sporea I, Martie A, Bota S, Sirli R, Popescu A, Danila M. Characterization of focal liver lesions using contrast enhanced ultrasound as a first line method: a large monocentric experience. J Gastrointestin Liver Dis. 2014;23:57-63.

32. Molins IG, Font JM, Alvaro JC, Navarro JL, Gil MF, Rodriguez CM. Contrast-enhanced ultrasound in diagnosis and characterization of focal hepatic lesions. World J Radiol. 2010;2:455-62. 
33. Chang CY, Hernandez-Prera JC, Roayaie S, Schwartz M, Thung SN. Changing epidemiology of hepatocellular adenoma in the United States: review of the literature. Int $J$ Hepatol. 2013;2013:604860.

34. Catalano O, Sandomenico F, Raso MM, Siani A. Low mechanical index contrast-enhanced sonographic findings of pyogenic hepatic abscesses. AJR Am J Roentgenol. 2004;182:447-50.

35. Kim TK, Jang HJ, Wilson SR. Benign liver masses: imaging with microbubble contrast agents. Ultrasound Q. 2006;22:31-9.

36. Kim TK, Lee KH, Khalili K, Jang HJ. Hepatocellular nodules in liver cirrhosis: contrast-enhanced ultrasound. Abdom Imaging. 2011;36:244-63.

37. Nordenstedt H, White DL, El-Serag HB. The changing pattern of epidemiology in hepatocellular carcinoma. Dig Liver Dis. 2010;42:S206-14.

38. Thakur S, Jhobta A, Dhiman DS, Sood RG, Chauhan A, Thakur CS. Role of contrast enhanced ultrasound in characterization of focal liver lesions. Egypt J Radiol Nucl Med. 2014;45:7-17.

39. Liu GJ, Xu HX, Lu MD, Xie XY, Xu ZF, Zheng YL, et al. Correlation between enhancement pattern of hepatocellular carcinoma on real-time contrast-enhanced ultrasound and tumour cellular differentiation on histopathology. Br J Radiol. 2007;80:321-30.

40. Takahashi M, Maruyama H, Ishibashi H, Yoshikawa M, Yokosuka O. Contrast-enhanced ultrasound with perflubutane microbubble agent: evaluation of differentiation of hepatocellular carcinoma. AJR Am J Roentgenol. 2011;196:W123-31.

41. Jang HJ, Yu H, Kim TK. Imaging of focal liver lesions. Semin Roentgenol. 2009;44:266-82.

42. Kim TK, Jang HJ. Contrast-enhanced ultrasound in the diagnosis of nodules in liver cirrhosis. World J Gastroenterol. 2014;20:3590-6.

43. Rossi S, Ghittoni G, Ravetta V, Torello Viera F, Rosa L, Serassi $\mathrm{M}$, et al. Contrast-enhanced ultrasonography and spiral computed tomography in the detection and characterization of portal vein thrombosis complicating hepatocellular carcinoma. Eur Radiol. 2008;18:1749-56.

44. Chen LD, Xu HX, Xie XY, Xie XH, Xu ZF, Liu GJ, et al. Intrahepatic cholangiocarcinoma and hepatocellular carcinoma: differential diagnosis with contrast-enhanced ultrasound. Eur Radiol. 2010;20:743-53.

45. Vilana R, Forner A, Bianchi L, Garcia-Criado A, Rimola J, de Lope CR, et al. Intrahepatic peripheral cholangiocarcinoma in cirrhosis patients may display a vascular pattern similar to hepatocellular carcinoma on contrast-enhanced ultrasound. Hepatology. 2010;51:2020-9.

46. Lassau N, Chami L, Chebil M, Benatsou B, Bidault S, Girard E, et al. Dynamic contrast-enhanced ultrasonography (DCE-US) and anti-angiogenic treatments. Discov Med. 2011;11:18-24.

47. Lassau N, Koscielny S, Chami L, Chebil M, Benatsou B, Roche A, et al. Advanced hepatocellular carcinoma: early evaluation of response to bevacizumab therapy at dynamic contrast-enhanced US with quantification-preliminary results. Radiology. 2011;258:291-300.

48. Pompili M, Riccardi L, Covino M, Barbaro B, Di Stasi C, Orefice $\mathrm{R}$, et al. Contrast-enhanced gray-scale harmonic ultrasound in the efficacy assessment of ablation treatment for hepatocellular carcinoma. Liver Int. 2005;25:954-61.

49. Solbiati L, Ierace T, Tonolini M, Cova L. Guidance and monitoring of radiofrequency liver tumor ablation with contrast-enhanced ultrasound. Eur J Radiol. 2004;51:S19-23.

50. Llovet JM, Real MI, Montana X, Planas R, Coll S, Aponte J, et al. Arterial embolisation or chemoembolisation versus symptomatic treatment in patients with unresectable hepatocellular carcinoma: a randomised controlled trial. Lancet. 2002;359:1734-9.

51. Lo CM, Ngan H, Tso WK, Liu CL, Lam CM, Poon RT, et al. Randomized controlled trial of transarterial lipiodol chemoembolization for unresectable hepatocellular carcinoma. Hepatology. 2002;35:1164-71.

52. Maruyama H, Yoshikawa M, Yokosuka O. Current role of ultrasound for the management of hepatocellular carcinoma. World J Gastroenterol. 2008;14:1710-9.

53. Xia Y, Kudo M, Minami Y, Hatanaka K, Ueshima K, Chung H, et al. Response evaluation of transcatheter arterial chemoembolization in hepatocellular carcinomas: the usefulness of sonazoid-enhanced harmonic sonography. Oncology. 2008;75:99-105.

54. Lencioni R, Llovet JM. Modified RECIST (mRECIST) assessment for hepatocellular carcinoma. Semin Liver Dis. 2010;30:52-60.

55. Wang Z, Tang J, An L, Wang W, Luo Y, Li J, et al. Contrastenhanced ultrasonography for assessment of tumor vascularity in hepatocellular carcinoma. $J$ Ultrasound Med. 2007;26:757-62.

56. Kim MY, Suk KT, Baik SK, Kim HA, Kim YJ, Cha SH, et al. Hepatic vein arrival time as assessed by contrast-enhanced ultrasonography is useful for the assessment of portal hypertension in compensated cirrhosis. Hepatology. 2012;56:1053-62.

57. Lim AK, Taylor-Robinson SD, Patel N, Eckersley RJ, Goldin RD, Hamilton G, et al. Hepatic vein transit times using a microbubble agent can predict disease severity non-invasively in patients with hepatitis C. Gut. 2005;54:128-33.

58. Cagini L, Gravante S, Malaspina CM, Cesarano E, Giganti M, Rebonato A, et al. Contrast enhanced ultrasound (CEUS) in blunt abdominal trauma. Crit Ultrasound J. 2013;5:S9.

59. Brown MA, Sirlin CB, Hoyt DB, Casola G. Screening ultrasound in blunt abdominal trauma. I Intensive Care Med. 2003;18:253-60.

60. Paajanen H, Lahti P, Nordback I. Sensitivity of transabdominal ultrasonography in detection of intraperitoneal fluid in humans. Eur Radiol. 1999;9:1423-5.

61. Poletti PA, Kinkel K, Vermeulen B, Irmay F, Unger PF, Terrier F. Blunt abdominal trauma: should US be used to detect both free fluid and organ injuries? Radiology. 2003;227:95-103.

62. Rothlin MA, Naf R, Amgwerd M, Candinas D, Frick T, Trentz O. Ultrasound in blunt abdominal and thoracic trauma. J Trauma. 1993;34:488-95.

63. Valentino M, Serra C, Zironi G, De Luca C, Pavlica P, Barozzi L. Blunt abdominal trauma: emergency contrast-enhanced sonography for detection of solid organ injuries. AJR Am J Roentgenol. 2006;186:1361-7.

64. Leen E, Ceccotti P, Moug SJ, Glen P, MacQuarrie J, Angerson 
WJ, et al. Potential value of contrast-enhanced intraoperative ultrasonography during partial hepatectomy for metastases: an essential investigation before resection? Ann Surg. 2006;243:236-40.

65. Lu Q, Luo Y, Yuan CX, Zeng Y, Wu H, Lei Z, et al. Value of contrast-enhanced intraoperative ultrasound for cirrhotic patients with hepatocellular carcinoma: a report of 20 cases. World $J$
Gastroenterol. 2008;14:4005-10.

66. Piscaglia F, Bolondi L, Italian Society for Ultrasound in M, Biology Study Group on Ultrasound Contrast A. The safety of Sonovue in abdominal applications: retrospective analysis of 23188 investigations. Ultrasound Med Biol. 2006;32:1369-75.

67. Lencioni R, Piscaglia F, Bolondi L. Contrast-enhanced ultrasound in the diagnosis of hepatocellular carcinoma. $J$ Hepatol. 2008;48:848-57. 